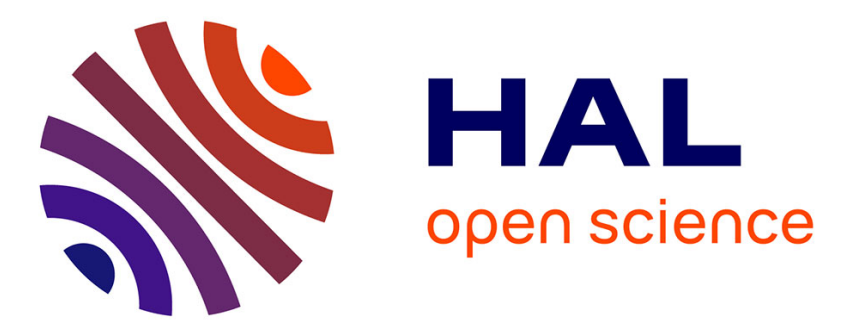

\title{
Stability analysis of sampled-data control systems under magnitude and rate saturating actuators
}

\author{
Alessandra H. K. Palmeira, João Manoel Gomes da Silva Jr, Sophie \\ Tarbouriech, I. M. F. Ghiggi
}

\section{- To cite this version:}

Alessandra H. K. Palmeira, João Manoel Gomes da Silva Jr, Sophie Tarbouriech, I. M. F. Ghiggi. Stability analysis of sampled-data control systems under magnitude and rate saturating actuators. 2015 European Control Conference (ECC), Jul 2015, Linz, Austria. pp.398-403, 10.1109/ECC.2015.7330576 . hal-01856299

\section{HAL Id: hal-01856299 \\ https://hal.laas.fr/hal-01856299}

Submitted on 10 Aug 2018

HAL is a multi-disciplinary open access archive for the deposit and dissemination of scientific research documents, whether they are published or not. The documents may come from teaching and research institutions in France or abroad, or from public or private research centers.
L'archive ouverte pluridisciplinaire HAL, est destinée au dépôt et à la diffusion de documents scientifiques de niveau recherche, publiés ou non, émanant des établissements d'enseignement et de recherche français ou étrangers, des laboratoires publics ou privés. 


\title{
Stability Analysis of Sampled-data Control Systems under Magnitude and Rate Saturating Actuators*
}

\author{
A. H. K. Palmeira ${ }^{1}$ and J. M. Gomes da Silva Jr. ${ }^{2}$ and S. Tarbouriech ${ }^{3}$ and I. M. F. Ghiggi ${ }^{4}$
}

\begin{abstract}
This paper addresses the problem of stability analysis of sampled-data control of linear systems in the presence of input magnitude and rate saturation. A position-type feedback modeling for the actuator is considered. Based on the use of a discrete-time quadratic Lyapunov function, a loopedfunctional and generalized sector relations (to cope with nested saturation functions), LMI conditions are derived to assess local (regional) and global stability of the sampled-data closed-loop systems under aperiodic sampling strategies.
\end{abstract}

\section{INTRODUCTION}

Actuator saturation is an ubiquitous feature in control systems. Due to physical or security limitations, the actuators are constrained to provide signals with both magnitude and rate (the derivative) inside certain intervals. The effective magnitude or rate saturation of the control signal is source of instability and performance degradation. Motivated by that, a large amount of works have been devoted to the study of the impact of control saturation on the behavior of closed-loop systems. Most of these works deal only with the problem of magnitude saturation (see for instance [1], [2], [3] and references therein). On the other hand, the rate of the control signal is also critical and deserves to be taken into account explicitly in many applications. This is the case in the aeronautic field, where the rate limitations in hydraulic actuators can cause the so-called pilot-inducedoscillations (PIO), which can lead to an effective disaster [4], [5]. Other examples are electromechanical actuators (such as valve positioners) and pneumatic actuators, which clearly present dynamic limitations.

Regarding the problem of stability analysis and stabilization of systems presenting magnitude (position) and rate saturation, many works can be cited. Basically, two approaches are followed. The first one considers the design of the controller (possibly nonlinear) in order

*This work was supported by CAPES, CNPQ (grants PQ 306210/2009-6, UNIV. 480638/2012-8), Brazil, and by ADNEC project STIC-AmSud grant number 13STIC-03

${ }^{1}$ Alessandra Helena Kimura Palmeira is with Department of Electrical Engineering, Federal University of Rio Grande do Sul, Porto Alegre, RS, Brazil, alessandrakimurap@gmail.com

${ }^{2}$ João Manoel Gomes da Silva Jr. is with Department of Electrical Engineering, Federal University of Rio Grande do Sul, Porto Alegre, RS, Brazil, jmgomes@ece.ufrgs.br

${ }^{3}$ Sophie Tarbouriech is with CNRS, LAAS, 7 avenue du Colonel Roche, F-31400, Toulouse, France, and Univ de Toulousse, LAAS, F-31400, Toulouse, France, tarbour@laas.fr

${ }^{4}$ Ilca Maria Ferrari Ghiggi is with IFSC-Campus Chapeco, SC, Brazil, ilcaghiggi@hotmail.com to generate a signal respecting the magnitude and rate bounds of the actuator [6], [7], [8]. The second approach considers a first-order model to represent the dynamics of the actuator, the so-called position-type feedback model. In this case, the saturation in rate appears as the saturation of the actuator state and when the time-constant of the first-order model tends to zero the "ideal" rate limiter is recovered [9]. Considering this approach we can cite works dealing with global [9], semi-global [10] and local (regional) stability [11]. Moreover, considering simultaneously rate and magnitude saturation leads to a system presenting two nested saturations. General results concerning systems with nested saturations can therefore be applied to consider the global or the regional stability or stabilization problems (see for instance [12], [13], [14]). It should be noted that the aforementioned references consider both the plant and the actuator model in continuous-time. Some counterpart results regarding discrete-time models can be found, for instance, in [8], [15].

On the other hand, regarding the case of sampled-data control in the presence of magnitude saturating actuators we can cite for instance [16] and [17]. However to the knowledge of the authors, there is a lack of works formally dealing with the problem of sampled-data control in the presence of rate saturation. In particular, it should be noticed that since the position-type feedback modeling is nonlinear (it presents a state saturation), the classical discretization strategies considering periodic sampling and the use of zero-order holders cannot be applied. Furthermore, the use of discretized models can be highly imprecise in this case. Another important aspect, which is quite related to the new paradigm of networked control [18], regards the possibility of aperiodic sampling.

This work aims somehow at filling this gap by providing a method to analyze the stability of sampleddata control-loops in the presence of magnitude and rate saturating actuators. We consider the position-type feedback modeling for the actuator and the possibility of having aperiodic sampling. In this context, conditions based on the use of a discrete-time Lyapunov function and a looped-functional [19] are derived to assess the stability of the closed-loop sampled-data system. The nested saturation nonlinearities resulting from the actuator modeling are taking into account by the application of generalized sector conditions as in [13]. The proposed conditions are in a quasi-LMI form (that is, if a scalar is fixed, LMI conditions are recovered) and can be easily 
incorporated in convex optimization problems aiming at maximizing estimates of the region of attraction of the origin or the maximum inter-sampling time for which the stability is ensured regionally or, when possible, globally. Notation. In this paper, the sets $\mathbb{N}, \mathbb{R}^{n}, \mathbb{R}^{n \times n}$ and $\mathbb{K}$ denote respectively the set of positive scalars, $n$ dimensional vectors, $n \times n$ matrices of real elements and the set of continuous functions from an interval $\left[0, T_{k}\right]$ to $\mathbb{R}^{n}$. The superscript ' $T^{\prime}$ stands for matrix transposition. $A_{(i)}$ denote the $i$ th row of matrix $A, A_{(i, j)}$ is its element in position $(i, j), H e\{A\}=A+A^{T}$. The symbols $I$ and 0 represent the identity and zero matrices of appropriate dimensions. The notation $A>($ or $<) 0$ means that $A$ is a symmetric and positive (or negative) definite matrix. The symbol $\star$ stands for symmetric blocks in a matrix. $\|\cdot\|$ denotes the Euclidean norm.

\section{Problem Statement}

The plant to be controlled is a linear continuous-time system described in state space as follows:

$$
\dot{x}_{p}(t)=A x_{p}(t)+B v(t),
$$

where $x_{p} \in \mathbb{R}^{n_{p}}$ and $v \in \mathbb{R}^{m}$ represent the vectors of plant state and input, respectively, and $A$ and $B$ are constant matrices of appropriate dimensions. In order to model the actuator constraints in position and rate, for each actuator we consider a first-order model [11] given by:

$$
\dot{x}_{a(i)}(t)=s a t_{r(i)}\left(-\lambda_{(i)} x_{a(i)}(t)+\lambda_{(i)} s a t_{p(i)}\left(u_{(i)}(t)\right)\right),
$$

for $i=1, \ldots, m . u \in \mathbb{R}^{m}$ and $x_{a} \in \mathbb{R}^{m}$ represent the input and the actuator state, respectively. The saturation functions are defined as follows

$$
\begin{aligned}
\operatorname{sat}_{p(i)}\left(\omega_{1(i)}\right) & =\operatorname{sign}\left(\omega_{1(i)}\right) \min \left\{\left|\omega_{1(i)}\right|, \bar{u}_{p(i)}\right\}, \\
\operatorname{sat}_{r(i)}\left(\omega_{2(i)}\right) & =\operatorname{sign}\left(\omega_{2(i)}\right) \min \left\{\left|\omega_{2(i)}\right|, \bar{u}_{r(i)}\right\},
\end{aligned}
$$

where $\bar{u}_{p(i)}$ and $\bar{u}_{r(i)}$ are the symmetrical position and rate bounds, respectively.

We consider that the control law is a sampled-data state feedback. The state is sampled at instant $t=t_{k}$ and the value of the control is kept constant, by means of zero-order hold $(\mathrm{ZOH})$, until the next sampling time, $t=$ $t_{k+1}$. In other words, $u(t)$ is given as follows:

$$
u(t)=u\left(t_{k}\right)=\left[\begin{array}{ll}
K_{p} & K_{a}
\end{array}\right]\left[\begin{array}{l}
x_{p}\left(t_{k}\right) \\
x_{a}\left(t_{k}\right)
\end{array}\right], \quad t_{k} \leq t<t_{k+1},
$$

where $t_{k}$ is the sampling instant, and $k \in \mathbb{N}$ is an increasing sequence of positive scalars. We suppose that the difference between two successive sampling instants, defined by $T_{k}=t_{k+1}-t_{k}$, satisfies $0 \leq \tau_{1} \leq T_{k} \leq \tau_{2}$.

Thus, the closed-loop system, issued from the connection between (1), (2) and (3), with $v(t)=x_{a}(t)$, can be described by:

$$
\begin{aligned}
\dot{x}_{p}(t)= & A x_{p}(t)+B x_{a}(t) \\
\dot{x}_{a}(t)= & \operatorname{sat}_{r}\left(-\Lambda x_{a}(t)+\Lambda s a t_{p}\left(K_{p} x_{p}\left(t_{k}\right)+K_{a} x_{a}\left(t_{k}\right)\right)\right) \\
& \text { for } t_{k} \leq t<t_{k+1}
\end{aligned}
$$

where $\Lambda \in \mathbb{R}^{m \times m}$ is a diagonal matrix with $\Lambda_{(i, i)}=\lambda_{(i)}$.
The model described by (4) can be rewritten using the generic model of systems with nested saturations [13], as follows:

$$
\begin{aligned}
& \dot{x}(t)=A_{2} x(t)+B_{2} \operatorname{sat}_{2}\left(A_{1} x(t)+B_{1} \operatorname{sat}_{1}(u(t))\right), \\
& u(t)=u\left(t_{k}\right)=C x\left(t_{k}\right), \quad t_{k} \leq t<t_{k+1},
\end{aligned}
$$

with the plant and actuator states represented by the augmented state vector $x(t)=\left[\begin{array}{cc}x_{p}(t)^{T} & x_{a}(t)^{T}\end{array}\right]^{T}, x \in \mathbb{R}^{n}$, $\operatorname{sat}_{1}(\cdot)=\operatorname{sat}_{p}(\cdot)$, with the bound $\bar{u}_{1}=\bar{u}_{p}, \operatorname{sat}_{2}(\cdot)=\operatorname{sat}_{r}(\cdot)$, with $\bar{u}_{2}=\bar{u}_{r}$, and

$$
\begin{gathered}
A_{2}=\left[\begin{array}{ll}
A & B \\
0 & 0
\end{array}\right], \quad B_{2}=\left[\begin{array}{l}
0 \\
I
\end{array}\right], \quad C=\left[\begin{array}{ll}
K_{p} & K_{a}
\end{array}\right], \\
A_{1}=\left[\begin{array}{ll}
0 & -\Lambda
\end{array}\right], \quad B_{1}=\Lambda .
\end{gathered}
$$

Define now $\chi_{k}(\tau)=x\left(t_{k}+\tau\right), \tau \in\left[0, T_{k}\right]$. Then, the closed-loop dynamics in the interval $\left[0, T_{k}\right]$ can be represented by:

$$
\dot{\chi}_{k}(\tau)=A_{2} \chi_{k}(\tau)+B_{2} \operatorname{sat}_{2}\left(A_{1} \chi_{k}(\tau)+B_{1} \operatorname{sat}_{1}\left(C \chi_{k}(0)\right)\right) .
$$

The set of all initial conditions $\left(\chi_{0}(0) \in \mathbb{R}^{n}\right)$, such that, the corresponding trajectories of the system (7) converge asymptotically to the origin corresponds to the so-called region of attraction of the origin $\left(\mathscr{R}_{a}\right)$. Due to the difficulty to determine analytically $\mathscr{R}_{a}$, a problem of interest is to compute an estimate $(\mathscr{D})$ of this region, such that, $\mathscr{D} \subset \mathscr{R}_{a} \subseteq \mathbb{R}^{n}$, taking into account both the actuator constraints and the fact that the interval between two successive sampling instants can vary.

The goal of this paper is to assess the local or global asymptotic stability of the origin of the sampled-data system (5) taking into account the actuator constraints and aperiodic sampling intervals $T_{k} \in\left[\tau_{1}, \tau_{2}\right]$. Then we focus on the following problems.

P1. Given the state feedback gains $K_{p}, K_{a}$ and the bounds $\tau_{1}$ and $\tau_{2}$ on $T_{k}$, maximize an estimate of region $\mathscr{R}_{a}$.

P2. Given the state feedback gains $K_{p}, K_{a}$, a set of admissible initial conditions $\left(\mathscr{X}_{0}\right)$ and $\tau_{1}$, maximize the upper bound on the interval between two successive sampling instants, $\tau_{2}$, such that the corresponding trajectories of the system converge asymptotically to the origin, provided that $x(0) \in \mathscr{X}_{0}$.

When the open-loop system is asymptotically stable, we will consider global stability assessment. In the global case, $\mathscr{R}_{a}$ corresponds to the whole state space.

\section{Preliminaries}

Using the notation $\chi_{k}(\tau)$, the following vector-valued dead-zone functions can be defined from the magnitude and rate saturation:

$$
\begin{aligned}
\psi_{1}\left(\chi_{k}\right)=\psi_{k_{1}}= & \operatorname{sat}_{1}\left(C \chi_{k}(0)\right)-C \chi_{k}(0), \\
\psi_{2}\left(\chi_{k}\right)=\psi_{k_{2}}= & \operatorname{sat}_{2}\left(A_{1} \chi_{k}(\tau)+B_{1} \operatorname{sat}_{1}\left(C \chi_{k}(0)\right)\right) \\
& -\left(A_{1} \chi_{k}(\tau)+B_{1}\left(\psi_{k_{1}}+C \chi_{k}(0)\right)\right) .
\end{aligned}
$$

Then, $\forall \tau \in\left[0, T_{k}\right]$, the closed-loop system (5) can be rewritten, with the matrices defined in (6), as follows:

$$
\begin{aligned}
\dot{\chi}_{k}(\tau)= & \left(A_{2}+B_{2} A_{1}\right) \chi_{k}(\tau)+B_{2} B_{1} C \chi_{k}(0) \\
& +B_{2} B_{1} \psi_{k_{1}}+B_{2} \psi_{k_{2}} .
\end{aligned}
$$


Regarding dead-zone functions, we recall now the following Lemma, that provides a generalized sector condition.

Lemma 1: [2] Define the set $\mathscr{S}(\bar{u})=\left\{\omega \in \mathbb{R}^{m}, v \in\right.$ $\mathbb{R}^{m} ;\left|\omega_{(i)}-v_{(i)}\right| \leq \bar{u}_{(i)}$, for $\left.i=1, \ldots, m\right\}$. If $\omega$ and $v$ are elements of set $\mathscr{S}(\bar{u})$, then the dead-zone nonlinearity $\psi(\omega)$, defined as $\psi(\omega)=\operatorname{sat}(\omega)-\omega$, satisfies

$$
\psi(\omega)^{T} U(\psi(\omega)+v) \leq 0
$$

for any diagonal and positive definite matrix $U \in \mathbb{R}^{m \times m}$.

In order to apply Lemma 1 to the dead-zone functions $\psi_{k_{1}}$ and $\psi_{k_{2}}$, consider matrices $G_{1}, G_{21}$ and $G_{22} \in \mathbb{R}^{m \times n}$ and $G_{23} \in \mathbb{R}^{m \times m}$, and define the following sets:

$$
\begin{aligned}
\mathscr{S}\left(\bar{u}_{1}\right)= & \left\{\chi_{k}(0) \in \mathbb{R}^{n} ;\left|\left(C-G_{1}\right)_{(i)} \chi_{k}(0)\right| \leq \bar{u}_{1(i)},\right. \\
& \text { for } i=1, \ldots, m\}, \\
\mathscr{S}\left(\bar{u}_{2}\right)= & \left\{\left(\chi_{k}(0), \chi_{k}(\tau), \psi_{k_{1}}\right) \in \mathbb{R}^{n} \times \mathbb{R}^{n} \times \mathbb{R}^{m} ;\right. \\
& \mid\left(A_{1}-G_{21}\right)_{(i)} \chi_{k}(\tau)+\left(B_{1} C-G_{22}\right)_{(i)} \chi_{k}(0) \\
+ & \left.\left(B_{1}-G_{23}\right)_{(i)} \psi_{k_{1}} \mid \leq \bar{u}_{2(i)}, \text { for } i=1, \ldots, m\right\} .
\end{aligned}
$$

Then, if $\chi_{k}(0) \in \mathscr{S}\left(\bar{u}_{1}\right)$, and $\left(\chi_{k}(0), \chi_{k}(\tau), \psi_{k_{1}}\right) \in \mathscr{S}\left(\bar{u}_{2}\right)$, then the following inequalities are satisfied for any diagonal and positive definite matrices $U_{1}$ and $U_{2} \in \mathbb{R}^{m \times m}$ :

$$
\begin{gathered}
\psi_{k_{1}}^{T} U_{1}\left(\psi_{k_{1}}+G_{1} \chi_{k}(0)\right) \leq 0, \\
\psi_{k_{2}}^{T} U_{2}\left(\psi_{k_{2}}+G_{21} \chi_{k}(\tau)+G_{22} \chi_{k}(0)+G_{23} \psi_{k_{1}}\right) \leq 0 .
\end{gathered}
$$

Based on conditions (12) and (13), from a discrete-time and positive definite function $V\left(\chi_{k}\right)$ and a continuoustime looped-functional $W\left(\tau, \chi_{k}\right)$, we state now a Theorem that allows to conclude about the regional stability of the closed-loop system (5) in a level set of the function $V\left(\chi_{k}\right)$. This result is basically an extension of Theorem 1 in [17], to cope with position and rate saturation.

Theorem 1: Consider a function $V\left(\chi_{k}\right): \mathbb{R}^{n} \rightarrow \mathbb{R}^{+}$, such that

$$
V\left(\chi_{k}\right)>0, \forall \chi_{k} \neq 0,
$$

and a continuous-time functional $V_{0}:\left[0, \tau_{2}\right] \times \mathbb{K} \rightarrow \mathbb{R}$, which satisfies for all $\chi_{k} \in \mathbb{K}$

$$
\begin{gathered}
V_{0}\left(T_{k}, \chi_{k}\right)=V_{0}\left(0, \chi_{k}\right)=0 \\
\forall \tau \in\left(0, T_{k}\right), \quad V_{0}\left(\tau, \chi_{k}\right)>0 .
\end{gathered}
$$

Define now $W\left(\tau, \chi_{k}\right)=V\left(\chi_{k}\right)+V_{0}\left(\tau, \chi_{k}\right)$ and let $\dot{W}\left(\tau, \chi_{k}\right)$ be the time-derivative of $W\left(\tau, \chi_{k}\right)$ with respect to $\tau$, along the trajectories of the system (9), for $\tau \in\left[0, T_{k}\right]$ and $0<$ $\tau_{1} \leq T_{k} \leq \tau_{2}$. If there exist matrices $G_{1}, G_{21}, G_{22} \in \mathbb{R}^{m \times n}$, $G_{23} \in \mathbb{R}^{m \times m}$, diagonal and positive definite matrices $U_{1}$ and $U_{2} \in \mathbb{R}^{m \times m}$, such that, the following inequalities are satisfied

$$
\begin{gathered}
\left\|\left(C_{(i)}-G_{1(i)}\right) \chi_{k}(0)\right\|^{2} \leq \bar{u}_{1(i)}^{2} V\left(\chi_{k}(0)\right) \\
\left\|\left[\begin{array}{lll}
\Theta_{1(i)} & \Theta_{2(i)} & \Theta_{3(i)}
\end{array}\right]\left[\begin{array}{c}
\chi_{k}(\tau) \\
\chi_{k}(0) \\
\psi_{k_{1}}
\end{array}\right]\right\|^{2} \leq \bar{u}_{2(i)}^{2} V\left(\chi_{k}(\tau)\right),
\end{gathered}
$$

$$
\begin{gathered}
\dot{W}\left(\tau, \chi_{k}\right)-2 \psi_{k_{1}}^{T} U_{1}\left[\psi_{k_{1}}+G_{1} \chi_{k}(0)\right]-2 \psi_{k_{2}}^{T} U_{2}\left[\psi_{k_{2}}\right. \\
\left.+G_{21} \chi_{k}(\tau)+G_{22} \chi_{k}(0)+G_{23} \psi_{k_{1}}\right]<0,
\end{gathered}
$$

for $i=1, \ldots, m$ and with $\Theta_{1}=A_{1}-G_{21}, \Theta_{2}=B_{1} C-G_{22}$ and $\Theta_{3}=B_{1}-G_{23}$. Then for any initial condition $x(0)=\chi_{0}(0)$ in the set

$$
\mathscr{D}=\left\{\chi_{0} \in \mathbb{R}^{n} ; V\left(\chi_{0}\right) \leq 1\right\}
$$

it follows that:

a) $\Delta V\left(\chi_{k}\right)=V\left(\chi_{k}\left(T_{k}\right)\right)-V\left(\chi_{k}(0)\right)<0$,

b) the corresponding trajectories of the sampled-data system (5) with $T_{k} \in\left[\tau_{1}, \tau_{2}\right]$ converge asymptotically to the origin.

Proof: See appendix.

In the case the open-loop system (1) is asymptotically stable, the following Corollary provides a condition to assess the global asymptotically stability of the origin.

Corollary 1: Consider a function $V\left(\chi_{k}\right): \mathbb{R}^{n} \rightarrow \mathbb{R}^{+}$, such that, $V\left(\chi_{k}\right)>0, \forall \chi_{k} \neq 0$, and a continuous-time functional $V_{0}:\left[0, \tau_{2}\right] \times \mathbb{K} \rightarrow \mathbb{R}$, which satisfies for all $\chi_{k} \in$ $\mathbb{K}, \forall T_{k} \in\left[\tau_{1}, \tau_{2}\right], V_{0}\left(T_{k}, \chi_{k}\right)=V_{0}\left(0, \chi_{k}\right)=0$.

Define now $W\left(\tau, \chi_{k}\right)=V\left(\chi_{k}\right)+V_{0}\left(\tau, \chi_{k}\right)$ and let $\dot{W}\left(\tau, \chi_{k}\right)$ be the time-derivative of $W\left(\tau, \chi_{k}\right)$ with respect to $\tau$, along the trajectories of the system (9), for $\tau \in\left[0, T_{k}\right]$ and $0<$ $\tau_{1} \leq T_{k} \leq \tau_{2}$. If there exist diagonal and positive definite matrices $U_{1}$ and $U_{2} \in \mathbb{R}^{m \times m}$, such that the following inequality is satisfied

$$
\begin{gathered}
\dot{W}\left(\tau, \chi_{k}\right)-2 \psi_{k_{1}}^{T} U_{1}\left[\psi_{k_{1}}+C \chi_{k}(0)\right]-2 \psi_{k_{2}}^{T} U_{2}\left[\psi_{k_{2}}\right. \\
\left.+A_{1} \chi_{k}(\tau)+B_{1} C \chi_{k}(0)+B_{1} \psi_{k_{1}}\right]<0
\end{gathered}
$$

Then, it follows that the origin of the system (5) is globally asymptotically stable, for $T_{k} \in\left[\tau_{1}, \tau_{2}\right]$.

\section{Asymptotic Stability of SATURATED AND SAMPLED-DATA SYSTEMS}

In this section, LMI conditions for regional and global asymptotic stability analysis of the sampled-data system (5) are derived from Theorem 1 and Corollary 1, considering appropriate choices for $V\left(\chi_{k}\right)$ and $V_{0}\left(\tau, \chi_{k}\right)$.

Theorem 2: Consider the sampled-data system (5) with $T_{k} \in\left[\tau_{1}, \tau_{2}\right], 0<\tau_{1} \leq \tau_{2}$. If there exist symmetric and positive definite matrices $\tilde{P}, \tilde{S}_{1}$ and $\tilde{R} \in \mathbb{R}^{n \times n}$, diagonal and positive definite matrices $\tilde{U}_{1}$ and $\tilde{U}_{2} \in \mathbb{R}^{m \times m}$, a symmetric and positive definite matrix $\tilde{X}_{1} \in \mathbb{R}^{(m+n) \times(m+n)}$, matrices $\tilde{Y} \in \mathbb{R}^{n \times n}, \tilde{G}_{1}$ and $\tilde{G}_{21} \in \mathbb{R}^{m \times n}, \tilde{N} \in \mathbb{R}^{(3 n+2 m) \times n}$ and a positive scalar $\varepsilon$, that satisfy the following inequalities, for $j=1,2$ and $i=1, \ldots, m$ :

$$
\begin{gathered}
\tilde{\Pi}_{1}+\tau_{j} \tilde{\Pi}_{2}+\tau_{j} \tilde{\Pi}_{3}<0, \\
{\left[\begin{array}{cc}
\tilde{\Pi}_{1}-\tau_{j} \tilde{\Pi}_{3} & \tau_{j} \tilde{N} \\
\star & -\tau_{j} \tilde{R}
\end{array}\right]<0,} \\
{\left[\begin{array}{cc}
\tilde{P} & \left(C \tilde{Y}-\tilde{G}_{1}\right)_{(i)}^{T} \\
\star & \bar{u}_{1(i)}^{2}
\end{array}\right] \geq 0,} \\
{\left[\begin{array}{cc}
\tilde{P} & \left(A_{1} \tilde{Y}-\tilde{G}_{21}\right)_{(i)}^{T} \\
\star & \bar{u}_{2(i)}^{2}
\end{array}\right] \geq 0,}
\end{gathered}
$$


with

$$
\begin{aligned}
\tilde{\Pi}_{1}= & H e\left\{M_{1}^{T} \tilde{P} M_{3}-M_{4}^{T} \tilde{G}_{1} M_{2}-M_{5}^{T} \tilde{G}_{21} M_{1}\right. \\
& -M_{5}^{T} B_{1} C \tilde{Y} M_{2}-M_{5}^{T} B_{1} \tilde{U}_{1} M_{4}-\tilde{N} M_{12} \\
& +\left(\varepsilon M_{1}^{T}+M_{3}^{T}\right)\left(\left(A_{2}+B_{2} A_{1}\right) \tilde{Y} M_{1}\right. \\
& +B_{2} B_{1} C \tilde{Y} M_{2}-\tilde{Y} M_{3}+B_{2} B_{1} \tilde{U}_{1} M_{4} \\
& \left.\left.+B_{2} \tilde{U}_{2} M_{5}\right)\right\}-2 M_{4}^{T} \tilde{U}_{1} M_{4} \\
& -2 M_{5}^{T} \tilde{U}_{2} M_{5}-M_{12}^{T} \tilde{S}_{1} M_{12}, \\
\tilde{\Pi}_{2}= & H e\left\{M_{3}^{T} \tilde{S}_{1} M_{12}\right\}+M_{3}^{T} \tilde{R} M_{3}, \\
\tilde{\Pi}_{3}= & M_{24}^{T} \tilde{X}_{1} M_{24},
\end{aligned}
$$

and auxiliary matrices of appropriate dimensions

$$
\begin{array}{lll}
M_{1}=\left[\begin{array}{lllll}
I & 0 & 0 & 0 & 0
\end{array}\right], & M_{2}=\left[\begin{array}{lllll}
0 & I & 0 & 0 & 0
\end{array}\right], \\
M_{3}=\left[\begin{array}{llllll}
0 & 0 & I & 0 & 0
\end{array}\right], & M_{4}=\left[\begin{array}{lllll}
0 & 0 & 0 & I & 0
\end{array}\right], \\
M_{5}=\left[\begin{array}{lllll}
0 & 0 & 0 & 0 & I
\end{array}\right], & M_{12}=M_{1}-M_{2}, & \\
M_{24}=\left[\begin{array}{lll}
M_{2}^{T} & M_{4}^{T}
\end{array}\right]^{T} . & &
\end{array}
$$

Then, for any initial condition $x(0)=\chi_{0}(0)$ belonging to the set

$$
\mathscr{E}(P)=\left\{x \in \mathbb{R}^{n} ; x^{T} P x \leq 1\right\},
$$

where $P=\tilde{Y}^{-T} \tilde{P} \tilde{Y}^{-1}$, the corresponding trajectories of the sampled-data system (5) converge asymptotically to the origin.

Proof: See appendix.

Based on the result stated in Corollary 1, the following Corollary provides a condition to assess the global asymptotically stability of the origin of the sampled-data system (5).

Corollary 2: Consider the sampled-data system (5) with $T_{k} \in\left[\tau_{1}, \tau_{2}\right], 0<\tau_{1} \leq \tau_{2}$. If there exist symmetric and positive definite matrices $\tilde{P}, \tilde{S}_{1}$ and $\tilde{R} \in \mathbb{R}^{n \times n}$, diagonal and positive definite matrices $\tilde{U}_{1}$ and $\tilde{U}_{2} \in \mathbb{R}^{m \times m}$, symmetric and positive definite matrix $\tilde{X}_{1} \in \mathbb{R}^{(m+n) \times(m+n)}$, matrices $\tilde{N} \in \mathbb{R}^{(3 n+2 m) \times n}$ and $\tilde{Y} \in \mathbb{R}^{n \times n}$ and a positive scalar $\varepsilon$, such that, the matrix inequalities (21), (22) are satisfied, with $\tilde{G}_{1}=C \tilde{Y}$ and $\tilde{G}_{21}=A_{1} \tilde{Y}$, then, the origin is globally asymptotically stable.

\section{Numerical and Optimization Issues}

Based on the conditions given in Theorem 2 and Corollary 2, we propose now optimization problems to solve Problems P1 and P2 stated in Section II. We first recall the following result.

Lemma 2: [17] Let $P$ be a positive definite matrix and define $\tilde{P}=M^{T} P M$, with $M$ being a non-singular matrix. If

$$
\left[\begin{array}{cc}
P_{0} & I \\
I & M+M^{T}-\tilde{P}
\end{array}\right]>0
$$

then, $P<P_{0}$.

\section{A. Maximization of the estimate of $\mathscr{R}_{a}$}

Note that the set $\mathscr{E}(P)$ provided by the satisfaction of the conditions in Theorem 2 is, by definition, included in $\mathscr{R}_{a}$ and can be used as an estimate of it. Then, from Problem P1, given $T_{k} \in\left[\tau_{1}, \tau_{2}\right]$ and considering some size criterion, the goal is to maximize the set $\mathscr{E}(P)$. With this aim, we can for instance maximize the minor axis of $\mathscr{E}(P)$. This can be accomplished from the following optimization problem

$$
\begin{aligned}
& \min \delta \\
& \text { subject to } \\
& (21),(22),(23),(24), \\
& {\left[\begin{array}{cc}
\delta I & I \\
I & \tilde{Y}+\tilde{Y}^{T}-\tilde{P}
\end{array}\right]>0 .}
\end{aligned}
$$

From Lemma 2 and since $P=\tilde{Y}^{-T} \tilde{P} \tilde{Y}^{-1}$, the last inequality in (29) implies that $P<\delta I$. Then, the maximal eigenvalue of $P$ is smaller than $\delta$. Hence, the minimization of $\delta$ ensures the maximization of the minor axis of $\mathscr{E}(P)$. Note that, for $\tau_{1}$ and $\tau_{2}$ given, constraints in (29) are LMIs for a fixed $\varepsilon$. Then the optimal solution of (29) can be obtained by solving LMI-based problems on a grid in $\varepsilon$.

\section{B. Maximization of the sampling interval}

From Problem P2, we consider a region of admissible initial conditions for the sampled-data system (5) given in the following form

$$
\mathscr{E}\left(P_{0}\right)=\left\{x \in \mathbb{R}^{n} ; x^{T} P_{0} x \leq 1\right\} \text {, with } P_{0}=P_{0}^{T}>0 .
$$

Then for $\tau_{1}$ given, the goal is to find the maximum value of $\tau_{2}$, for which the stability is ensured for $T_{k} \in$ $\left[\tau_{1}, \tau_{2}\right]$. This can be accomplished from the following optimization problem

$$
\begin{aligned}
& \max \tau_{2} \\
& \text { subject to } \\
& (21),(22),(23),(24), \\
& {\left[\begin{array}{cc}
P_{0} & I \\
I & \tilde{Y}+\tilde{Y}^{T}-\tilde{P}
\end{array}\right]>0 .}
\end{aligned}
$$

Note that, from Lemma 2 , the last inequality implies that $P<P_{0}$ and $\mathscr{E}\left(P_{0}\right) \subseteq \mathscr{E}(P)$. Hence, considering $\tau_{1}$ given, the optimization problem (31) can be solved as a feasibility LMI problem by iteratively increasing $\tau_{2}$ and testing the feasibility of LMIs, for a fixed $\varepsilon$.

On the other hand, if the open-loop system is asymptotically stable, we can attempt to find the maximal bound $\tau_{2}$ on the sampling interval for which the global stability of the sampled-data system (5) is ensured. The following optimization problem can be used in this case

$$
\begin{aligned}
& \max \tau_{2} \\
& \text { subject to } \\
& (21),(22)
\end{aligned}
$$

with $\tilde{G}_{1}=C \tilde{Y}$ and $\tilde{G}_{21}=A_{1} \tilde{Y}$.

\section{Illustrative EXAMPLE}

Consider the numerical example borrowed from [11], where system (4) is described by the following matrices

$$
\begin{gathered}
A=\left[\begin{array}{cc}
0 & 1 \\
10 & -0.1
\end{array}\right] \quad B=\left[\begin{array}{l}
0 \\
1
\end{array}\right] \quad \Lambda=20 \\
C=\left[\begin{array}{lll}
-6.4276 & -2 & 0.4598
\end{array}\right] .
\end{gathered}
$$

with the bounds of magnitude and rate saturation being $\bar{u}_{1}=1$ and $\bar{u}_{2}=10$. By considering $T_{k} \in[0.01,0.06]$ and 
the optimization problem (29), for $\varepsilon=3$, we obtain an estimate of the region of the attraction defined with

$$
P=\left[\begin{array}{ccc}
567.3811 & 176.6072 & 28.8366 \\
176.6072 & 54.9722 & 8.9760 \\
28.8366 & 8.9760 & 2.1313
\end{array}\right] \text {. }
$$

Considering the initial condition $x(0)=$ $\left[\begin{array}{lll}0.16 & -0.74 & 1.2\end{array}\right]^{T} \in \mathscr{E}(P)$ and an aperiodic sampling with $T_{k} \in[0.01,0.06]$, simulation results are shown in Figures 1 and 2. Figure 1 depicts the signals $\operatorname{sat}_{p}(u)$ and $\dot{x}_{a}$, Figure 2 presents the plant states, the actuator state $x_{a}$ and $\operatorname{sat}_{p}(u)$.

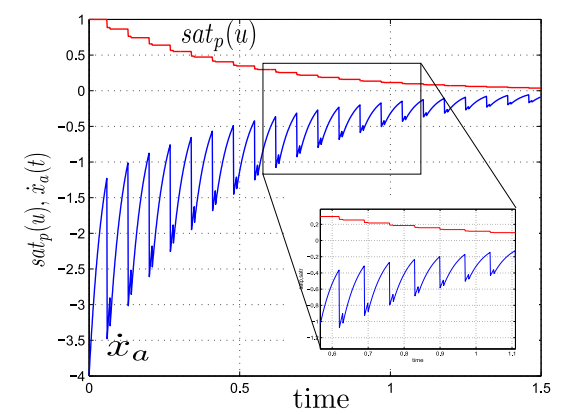

Fig. 1. Signals $\operatorname{sat}_{p}(u)$ (in red) and $\dot{x}_{a}$ (in blue), for $x(0)=[0.16-$ $0.741 .2]^{T}$

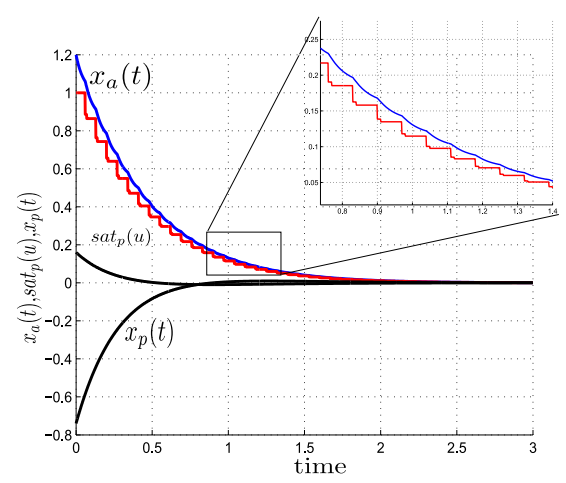

Fig. 2. Plant states (in black), actuator state (in blue) and $s_{t}(u)$ (in red), for $x(0)=\left[\begin{array}{lll}0.16 & -0.74 & 1.2\end{array}\right]^{T}$

Table I shows the trade-off between the size of the sampling interval $T_{k}=\left[\tau_{1}, \tau_{2}\right]$ and the size of the estimate of the region of attraction $\mathscr{E}(P)$ obtained from the optimization problem (29). We consider a fixed value for $\tau_{1}\left(\tau_{1}=0.01\right)$ and different values for $\tau_{2}$. As $\tau_{2}$ increases, i.e., larger is the interval $\left[\tau_{1}, \tau_{2}\right]$, larger values for $\delta$ are obtained, i.e., smaller is $\mathscr{E}(P)$. Note that the case of constant sampling periods $\left(\tau_{1}=\tau_{2}\right)$ leads to a larger set when compared to the cases of aperiodic sampling.

\section{Conclusion}

In this paper conditions for the stability analysis of sampled-data linear control systems with actuators presenting both magnitude and rate saturation have been proposed. The control signal has been assumed to be constant between two successive sampling instants and
TABLE I

Values of $\delta$ And trace of $P$, With Fixed $\tau_{1}=0.01$ FOR DIFFERENT VALUES OF $\tau_{2}$.

\begin{tabular}{|c|c|c|}
\hline$\tau_{2}$ & $\delta \times\left(10^{2}\right)$ & $\operatorname{trace}(P) \times\left(10^{2}\right)$ \\
\hline \hline $1 \times \tau_{1}$ & 4.82 & 4.81 \\
\hline $2 \times \tau_{1}$ & 4.93 & 4.91 \\
\hline $3 \times \tau_{1}$ & 5.06 & 5.04 \\
\hline $4 \times \tau_{1}$ & 5.26 & 5.22 \\
\hline $5 \times \tau_{1}$ & 6.24 & 5.85 \\
\hline $6 \times \tau_{1}$ & 8.04 & 6.36 \\
\hline
\end{tabular}

the continuous behavior of the plant and the nonlinear actuator model have been explicitly considered.

These conditions being LMIs for a fixed scalar $\varepsilon$, convex optimization problems are proposed to compute an estimate of the region of attraction or, given a set of admissible initial conditions, compute the maximal admissible inter-sampling time for which the convergence of the trajectories to the origin is ensured.

\section{APPENDIX}

\section{A. Proof of Theorem 1} that:

Suppose $\chi_{0}(0) \in \mathscr{D}$, then from (16) and (17), for $i=1, \ldots, m$, it follows

$$
\begin{gathered}
\chi_{0}(0)^{T}\left(C_{(i)}-G_{1(i)}\right)^{T}\left(C_{(i)}-G_{1(i)}\right) \chi_{0}(0) \leq \bar{u}_{1(i)}^{2} \\
{\left[\begin{array}{c}
\chi_{0}(0) \\
\chi_{0}(0) \\
\psi_{0_{1}}
\end{array}\right]^{T}\left[\begin{array}{c}
\Theta_{1(i)}^{T} \\
\Theta_{2(i)}^{T} \\
\Theta_{3(i)}^{T}
\end{array}\right]\left[\begin{array}{lll}
\Theta_{1(i)} & \Theta_{2(i)} & \Theta_{3(i)}
\end{array}\right]\left[\begin{array}{c}
\chi_{0}(0) \\
\chi_{0}(0) \\
\psi_{0_{1}}
\end{array}\right] \leq \bar{u}_{2(i)}^{2} .}
\end{gathered}
$$

It means that $\chi_{0}(0) \in \mathscr{S}\left(\bar{u}_{1}\right)$ and $\left(\chi_{0}(0), \chi_{0}(0), \psi_{0_{1}}\right) \in \mathscr{S}\left(\bar{u}_{2}\right)$. Hence, we conclude from (12), (13) and (18) that $\dot{W}\left(0, \chi_{0}(0)\right)<0$. Taking into account that $V_{0}\left(\tau, \chi_{k}\right)>0$, for $\tau \in\left(0, T_{k}\right)$ and $V_{0}\left(0, \chi_{k}\right)=V_{0}\left(T_{k}, \chi_{k}\right)=0$, it follows that $V\left(\chi_{k}\right) \leq W\left(\tau, \chi_{k}\right)$. This fact along with $W\left(0, \chi_{0}\right)<0$, leads to:

$V\left(\chi_{0}(\rho)\right) \leq W\left(\rho, \chi_{0}(\rho)\right)<W\left(0, \chi_{0}(0)\right)=V\left(\chi_{0}(0)\right)+V_{0}\left(0, \chi_{0}(0)\right)$, for $\rho \rightarrow 0^{+}$.

Since from $(15), V_{0}\left(0, \chi_{0}(0)\right)=0$, then we conclude that $V\left(\chi_{0}(\rho)\right)<$ $V\left(\chi_{0}(0)\right)$, i.e., $\chi_{0}(\rho) \in \mathscr{D}$. Repeating now the reasoning, we conclude that $V\left(\chi_{0}(\tau)\right)<V\left(\chi_{0}(0)\right), \forall \tau \in\left[0, T_{k}\right]$, i.e., $\left(\chi_{0}(\tau), \chi_{0}(0), \psi_{0_{1}}\right) \in \mathscr{S}\left(\bar{u}_{2}\right), \forall \tau \in\left[0, T_{k}\right]$, and we conclude that $W\left(\tau, \chi_{0}(\tau)\right)<0, \forall \tau \in\left[0, T_{k}\right]$.

Recalling that

$$
\dot{W}\left(\tau, \chi_{0}(\tau)\right)=\frac{d}{d \tau}\left(V\left(\chi_{0}(\tau)\right)+V_{0}\left(\tau, \chi_{0}(\tau)\right)\right)<0,
$$

and integrating $\dot{W}\left(\tau, \chi_{0}(\tau)\right)$ over the interval $\left[0, T_{k}\right]$ it follows that

$$
V\left(\chi_{0}\left(T_{k}\right)\right)-V\left(\chi_{0}(0)\right)<0 .
$$

Since $\chi_{k+1}(0)=\chi_{k}\left(T_{k}\right)$, this procedure can now be repeated and we conclude that $V\left(\chi_{k}\right)$ is decreasing at the sampling instants, i.e, $V\left(\chi_{k}\right)$ is a discrete-time Lyapunov function for the system (5).

\section{B. Proof of Theorem 2}

Consider a quadratic candidate Lyapunov function $V\left(\chi_{k}(\tau)\right)=$ $\chi_{k}(\tau)^{T} P \chi_{k}(\tau), \chi_{k} \in \mathbb{R}^{n}$, the matrix $P=P^{T}>0$, and a candidate continuoustime functional $V_{0}\left(\tau, \chi_{k}\right)$ defined for all $\tau \in\left[0, T_{k}\right]$, as follows

$$
\begin{aligned}
V_{0}\left(\tau, \chi_{k}\right)= & \left(T_{k}-\tau\right)\left\{\left[\chi_{k}(\tau)-\chi_{k}(0)\right]^{T} S_{1}\left[\chi_{k}(\tau)-\chi_{k}(0)\right]+\tau\left[\begin{array}{c}
\chi_{k}(0) \\
\psi_{k_{1}}
\end{array}\right]^{T} X_{1}\left[\begin{array}{c}
\chi_{k}(0) \\
\psi_{k_{1}}
\end{array}\right]\right. \\
& \left.+\int_{0}^{\tau} \dot{\chi}_{k}^{T}(\theta) R \dot{\chi}_{k}(\theta) d \theta\right\},
\end{aligned}
$$

with symmetric and positive definite matrices $X_{1} \in \mathbb{R}^{(m+n) \times(m+n)}, S_{1}$ and $R \in$ $\mathbb{R}^{n \times n}$. Note that, $V_{0}\left(\tau, \chi_{k}\right)$ satisfies conditions of Theorem 1, i.e., $V_{0}\left(0, \chi_{k}\right)=$ $V\left(T_{k}, \chi_{k}\right)=0$. Since for $\tau=0$, we have $\left[\chi_{k}(\tau)-\chi_{k}(0)\right]=0$, the integral term is equal to zero, and for $\tau=T_{k},\left(T_{k}-\tau\right)=0$.

From $V\left(\chi_{k}\right)$ and $V_{0}\left(\tau, \chi_{k}\right)$, define now $W\left(\tau, \chi_{k}\right)=V\left(\chi_{k}\right)+V_{0}\left(\tau, \chi_{k}\right)$ as in Theorem 1. Since $X_{1}, S_{1}, R>0, V_{0}\left(\tau, \chi_{k}\right)>0$ for any $\tau \in\left(0, T_{k}\right)$, then, $V\left(\chi_{k}(\tau)\right) \leq W\left(\tau, \chi_{k}\right)$. 
Define now $\Gamma\left(\tau, \chi_{k}\right)=\dot{W}\left(\tau, \chi_{k}\right)-2 \psi_{k_{1}}^{T} U_{1}\left[\psi_{k_{1}}+G_{1} \chi_{k}(0)\right]-2 \psi_{k_{2}}^{T} U_{2}\left[\psi_{k_{2}}+\right.$ $\left.G_{21} \chi_{k}(\tau)+G_{22} \chi_{k}(0)+G_{23} \psi_{k_{1}}\right]$, with $G_{22}=B_{1} C$ and $G_{23}=B_{1}$. It follows that

$$
\begin{aligned}
\Gamma\left(\tau, \chi_{k}\right)= & 2 \chi_{k}^{T}(\tau) P \dot{\chi}_{k}(\tau)-2 \psi_{k_{1}}^{T} U_{1}\left[\psi_{k_{1}}+G_{1} \chi_{k}(0)\right] \\
& -2 \psi_{k_{2}}^{T} U_{2}\left[\psi_{k_{2}}+G_{21} \chi_{k}(\tau)+B_{1} C \chi_{k}(0)+B_{1} \psi_{k_{1}}\right] \\
& +\left(T_{k}-\tau\right)\left\{\dot{\chi}_{k}(\tau)^{T}\left[R \dot{\chi}_{k}(\tau)+2 S_{1}\left(\chi_{k}(\tau)-\chi_{k}(0)\right)\right]\right. \\
& \left.-\left[\chi_{k}(\tau)-\chi_{k}(0)\right]^{T} S_{1}\left[\chi_{k}(\tau)-\chi_{k}(0)\right]\right\} \\
& +\left(T_{k}-2 \tau\right)\left[\begin{array}{c}
\chi_{k}(0) \\
\psi_{k_{1}}
\end{array}\right]^{T} X_{1}\left[\begin{array}{c}
\chi_{k}(0) \\
\psi_{k_{1}}
\end{array}\right]-\int_{0}^{\tau} \dot{\chi}_{k}^{T}(\theta) R \dot{\chi}_{k}(\theta) d \theta .
\end{aligned}
$$

We show now that the satisfaction of (21) and (22) implies that $\Gamma\left(\tau, \chi_{k}\right)<0$. Consider the following vector $\xi_{k}(\tau)=\xi_{k}=$

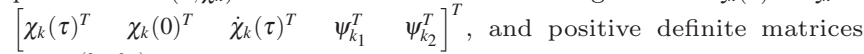
$N \in \mathbb{R}^{(3 n+2 m) \times n}$ and $R \in \mathbb{R}^{n \times n}$. Hence for the integral term of (35), the following inequality holds

$$
\int_{0}^{\tau} \dot{\chi}_{k}^{T}(\theta) R \dot{\chi}_{k}(\theta) d \theta-2 \xi_{k}^{T}(\tau) N\left[\chi_{k}(\tau)-\chi_{k}(0)\right]+\tau \xi_{k}^{T}(\tau) N R^{-1} N^{T} \xi_{k}(\tau) \geq 0 .
$$

On the other hand, from (9), it follows that $2\left(\chi_{k}^{T}(\tau) Y_{1}^{T}+\right.$ $\left.\dot{\chi}_{k}^{T}(\tau) Y_{2}^{T}\right)\left\{-\dot{\chi}_{k}(\tau)+\left(A_{2}+A_{1} B_{2}\right) \chi_{k}(\tau)+B_{2} B_{1} C \chi_{k}(0)+B_{2} B_{1} \psi_{k_{1}}+B_{2} \psi_{k_{2}}\right\}=0$, for any matrices $Y_{1}$ and $Y_{2} \in \mathbb{R}^{n \times n}$. Hence, using $\xi_{k}$ and the auxiliary matrices from (26) one gets

$$
\xi_{k}^{T}\left\{2\left(M_{1}^{T} Y_{1}^{T}+M_{3}^{T} Y_{2}^{T}\right) M_{0}\right\} \xi_{k}=0,
$$

with $M_{0}=\left[\begin{array}{lllll}A_{2}+B_{2} A_{1} & B_{2} B_{1} C & -I & B_{2} B_{1} & B_{2}\end{array}\right]$. Using (36), (37) and the auxiliary matrices defined in (26), it follows that

$$
\Gamma\left(\tau, \chi_{k}\right) \leq \xi_{k}^{T}\left[\Pi_{1}+\left(T_{k}-\tau\right) \Pi_{2}+\left(T_{k}-2 \tau\right) \Pi_{3}\right] \xi_{k}+\tau \xi_{k}^{T} N R^{-1} N^{T} \xi_{k},
$$

with $\Pi_{1}=H e\left\{M_{1}^{T} P M_{3}-M_{4}^{T} U_{1} M_{4}-M_{4}^{T} U_{1} G_{1} M_{2}-M_{5}^{T} U_{2} M_{5}-M_{5}^{T} U_{2} G_{21} M_{1}-\right.$ $\left.M_{5}^{T} U_{2} B_{1} C M_{2}-M_{5}^{T} U_{2} B_{1} M_{4}-N M_{12}+\left(M_{1}^{T} Y_{1}^{T}+M_{3}^{T} Y_{2}^{T}\right) M_{0}\right\}-M_{12}^{T} S_{1} M_{12}, \quad \Pi_{2}=$ $M_{3}^{T} R M_{3}+H e\left\{M_{3}^{T} S_{1} M_{12}\right\}$ and $\Pi_{3}=M_{24}^{T} X_{1} M_{24}$.

Consider now the contribution corresponding to terms of the form $\dot{\chi}_{k}^{T}(\tau)(.) \dot{\chi}_{k}(\tau)$ in the right hand side of (38), which is given by

$$
\begin{aligned}
& M_{3}\left[\Pi_{1}+\left(T_{k}-\tau\right) \Pi_{2}+\left(T_{k}-2 \tau\right) \Pi_{3}+\tau N R^{-1} N^{T}\right] M_{3}^{T} \\
& =-Y_{2}-Y_{2}^{T}+\left(T_{k}-\tau\right) R+\tau N_{3} R^{-1} N_{3}^{T},
\end{aligned}
$$

where $N_{3} \in \mathbb{R}^{n \times n}$ is a component of $N$. For a negative contribution of the term $\dot{\chi}_{k}^{T}(\tau)(.) \dot{\chi}_{k}(\tau), Y_{2}$ should be a non-singular matrix. Then we can define the matrices $\tilde{Y}=Y_{2}^{-1}, \tilde{U}_{1}=U_{1}^{-1}, \tilde{U}_{2}=U_{2}^{-1}$ and $\Xi=\operatorname{diag}\left\{\tilde{Y}, \tilde{Y}, \tilde{Y}, \tilde{U}_{1}, \tilde{U}_{2}\right\}$ and the relation $Y_{1}=\varepsilon Y_{2}$, for a positive scalar $\varepsilon$. Thus by considering $\tilde{\xi}_{k}=\Xi^{-1} \xi_{k}$, the inequality (38) is rewritten as

$\Gamma\left(\tau, \chi_{k}\right) \leq \tilde{\xi}_{k}^{T}\left[\Xi^{T} \Pi_{1} \Xi+\left(T_{k}-\tau\right) \Xi^{T} \Pi_{2} \Xi+\left(T_{k}-2 \tau\right) \Xi^{T} \Pi_{3} \Xi+\tau \Xi^{T} N R^{-1} N^{T} \Xi\right] \tilde{\xi}_{k}$.

From the auxiliary matrices defined in (26), we have

$$
\begin{aligned}
& \begin{array}{ccc}
M_{1} \Xi=\tilde{Y} M_{1} & M_{2} \Xi=\tilde{Y} M_{2} & M_{3} \Xi=\tilde{Y} M_{3} \\
M_{4} \Xi=\tilde{U}_{1} M_{4} & M_{5} \Xi=\tilde{U}_{2} M_{5} & M_{12} \Xi=\tilde{Y} M_{12}
\end{array} \quad M_{24} \Xi=\left[\begin{array}{cr}
\tilde{Y} & 0 \\
0 & \tilde{U}_{1}
\end{array}\right] M_{24} \\
& M_{0} \Xi=\left(A_{2}+B_{2} A_{1}\right) \tilde{Y} M_{1}+B_{2} B_{1} C \tilde{Y} M_{2}-\tilde{Y} M_{3}+B_{2} B_{1} \tilde{U}_{1} M_{4}+B_{2} \tilde{U}_{2} M_{5}
\end{aligned}
$$

Considering now the following change of variables

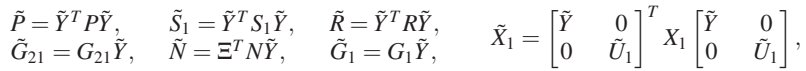

and applying in (39), with $\tilde{\Pi}_{1}, \tilde{\Pi}_{2}$ and $\tilde{\Pi}_{3}$ defined as in (25), it follows that

$$
\Gamma\left(\tau, \chi_{k}\right) \leq \tilde{\xi}_{k}^{T}\left[\tilde{\Pi}_{1}+\left(T_{k}-\tau\right) \tilde{\Pi}_{2}+\left(T_{k}-2 \tau\right) \tilde{\Pi}_{3}+\tau \tilde{N} R^{-1} \tilde{N}^{T}\right] \tilde{\xi}_{k}
$$

Hence if

$$
\tilde{\Pi}_{1}+\left(T_{k}-\tau\right) \tilde{\Pi}_{2}+\left(T_{k}-2 \tau\right) \tilde{\Pi}_{3}+\tau \tilde{N} \tilde{R}^{-1} \tilde{N}^{T} \leq 0,
$$

$\forall \tau \in\left[0, T_{k}\right]$, it follows that $\Gamma\left(\tau, \chi_{k}\right)<0$.

By convexity, (40) is satisfied $\forall \tau \in\left[0, T_{k}\right]$ and $T_{k} \in\left[\tau_{1}, \tau_{2}\right]$, if the following inequalities are verified

$$
\begin{gathered}
\tilde{\Pi}_{1}+\tau_{j} \tilde{\Pi}_{2}+\tau_{j} \tilde{\Pi}_{3}<0, \\
\tilde{\Pi}_{1}-\tau_{j} \tilde{\Pi}_{3}+\tau_{j} \tilde{N} \tilde{R}^{-1} \tilde{N}^{T}<0 .
\end{gathered}
$$

for $j=1,2$. Note that (21) is directly (41) and, by applying the Schur's complement, (22) is equivalent to (42). Thus, we conclude that (21) and (22) ensure that $\Gamma\left(\tau, \chi_{k}\right)<0$, which corresponds to (18) in Theorem 1.

Now from Theorem 1, in order to conclude that the trajectories converge to origin asymptotically provided $x(0) \in \mathscr{E}(P)$, we need to show that relations (16) and (17) are satisfied. By right and left-multiplying (23) and $(24)$ by $\operatorname{diag}\left\{\tilde{Y}^{-1}, I\right\}$, it follows that

$$
\chi_{k}(0)^{T}\left\{P-\frac{1}{\bar{u}_{1(i)}^{2}}\left(C_{(i)}-G_{1(i)}\right)^{T}\left(C_{(i)}-G_{1(i)}\right)\right\} \chi_{k}(0) \geq 0,
$$

i.e. $\chi_{k}(0)^{T}\left(C_{(i)}-G_{1(i)}\right)^{T}\left(C_{(i)}-G_{1(i)}\right) \chi_{k}(0) \leq V\left(\chi_{k}(0)\right) \bar{u}_{1(i)}^{2}$, and

$$
\chi_{k}(\tau)^{T}\left\{P-\frac{1}{\bar{u}_{2(i)}^{2}}\left(A_{1(i)}-G_{21(i)}\right)^{T}\left(A_{1(i)}-G_{21(i)}\right)\right\} \chi_{k}(\tau) \geq 0,
$$

i.e. $\chi_{k}(\tau)^{T}\left(A_{1(i)}-G_{21(i)}\right)^{T}\left(A_{1(i)}-G_{21(i)}\right) \chi_{k}(\tau) \leq V\left(\chi_{k}(\tau)\right) \bar{u}_{2(i)}^{2}$, where, in particular, we considered $G_{22}=B_{1} C$ and $G_{23}=B_{1}$ in the condition (13). That concludes the proof.

\section{REFERENCES}

[1] T. Hu and Z. Lin, Control systems with actuator saturation: analysis and design. Birkhauser, 2001.

[2] S. Tarbouriech, G. Garcia, J. M. Gomes da Silva Jr., and I. Queinnec, Stability and Stabilization of Linear Systems with Saturating Actuators, 1st ed. London: Springer, 2011.

[3] L. Zaccarian and A. R. Teel, Modern anti-windup synthesis. Princeton University Press, 2011.

[4] R. B. Miller and M. Pachter, "Maneuvering flight control with actuator constraints," Journal of Guidance, Control and Dynamics, vol. 20, no. 4, pp. 22-23, July-august 1997.

[5] L. Rundqwist and R. Hillgren, "Phase compensation of rate limiters in jas 39 gripen," in AIAA, 1996, pp. 69-79.

[6] V. Kapila and W. Haddad, "Fixed structue controller design with amplitude and rate nonlinearities," in Proc. of the 37th IEEE Conference on Decision and Control, Tampa (USA), 1998, pp. $3920-3921$.

[7] V. Kapila, H. Pan, and M. S. de Queiroz, "LMI-based control of linear systems with actuator amplitude and rate nonlinearities," in Proc. of the 38th IEEE Conference on Decision and Control, Phoenix, Arizona, 1999.

[8] J. M. Gomes da Silva Jr., D. Limon, T. Alamo, and E. Camacho, "Dynamic output feedback for discrete-time systems under amplitude and rate actuator constraints," IEEE Transactions on Automatic Control, vol. 50, pp. 2367-2372, Nov. 2008.

[9] A. Stoorvogel and A. Saberi, "Output regulation for linear systems to amplitude plus rate saturating actuators," Int. J. of Robust and Nonlinear Control, vol. 9, no. 10, pp. 631-657, 1999.

[10] Z. Lin, "Semi-global stabilization of linear systems with position and rate-limited actuators," System \& Control Letters, vol. 30, pp. 1-11, 1997.

[11] J. M. Gomes da Silva Jr., S. Tarbouriech, and G. Garcia, "Local stabilization of linear systems under amplitude and rate saturation actuators," IEEE Transctions on Automatic Control, vol. 48, no. 5, pp. 842-847, 2003.

[12] A. Bateman and Z. Lin, "An analysis and design method for linear systems under nested saturation," Systems 83 Control Letters, vol. 48, pp. 41-52, 2002.

[13] S. Tarbouriech, C. Prieur, and J. M. Gomes da Silva Jr., "Stability analysis and stabilization of systems presenting nested saturations," IEEE Transctions on Automatic Control, vol. 51, no. 8, pp. 1364-1371, 2006.

[14] B. Zhou, W. Zheng, and G. Duan, "An improved treatment of saturation nonlinearity with its application to control of systems subject to nested saturation," Automatica, vol. 47, no. 2, pp. 306-315, 2011.

[15] A. Bateman and Z. Lin, "An analysis and design method for discrete-time linear systems under nested saturation," IEEE Transctions on Automatic Control, vol. 47, no. 8, pp. 13051310, 2002.

[16] E. Fridman, A. Seuret, and J.-P. Richard, "Robust sampleddata stabilization of linear systems: an input delay approach," Automatica, vol. 40, pp. 1441-1446, 2004.

[17] A. Seuret and J. M. Gomes da Silva Jr., "Taking into acccount period variations and actuator saturation in sampled-data systems," Systems 86 Control Letters, vol. 61, pp. 1286-1293, 2012.

[18] W. Zhang, M. Branicky, and S. Phillips, "Stability of networked control systems," IEEE Control Systems Magazine, vol. 21, pp. 84-99, 2001.

[19] A. Seuret, "A novel stability anlysis of linear systems under asynchronous samplings," Automatica, vol. 48, pp. 177-182, 2012 . 